\title{
Early intervention in the treatment of rheumatoid arthritis: focus on tocilizumab
}

This article was published in the following Dove Press journal:

Therapeutics and Clinical Risk Management

24 October 2013

Number of times this article has been viewed

\section{Sedat Yilmaz \\ Ismail Simsek}

Division of Rheumatology, Gulhane School of Medicine, Ankara, Turkey
Correspondence: Ismail Simsek

Gulhane School of Medicine, Division of Rheumatology, 06018 Etlik, Ankara, Turkey $\mathrm{Tel}+903123043965$

Fax +903123043960

Email isimsekI@yahoo.com
Abstract: Tocilizumab is a fully humanized monoclonal antibody against interleukin-6 receptors that was approved for the treatment of patients with rheumatoid arthritis (RA). Several lines of evidence, obtained both from conventional disease-modifying anti-rheumatic drugs (DMARDs) and tumor necrosis factor (TNF) inhibitors, have supported the concept of "window of opportunity" as showing that these therapies consistently work better in early disease as compared to established RA. This review addresses the question of whether a window of opportunity gained with conventional DMARDs and TNF inhibitors can also be achieved with tocilizumab. To this end, data regarding the use of tocilizumab in early RA patients are summarized. Currently available data suggest that the earlier the treatment with tocilizumab, the better the clinical outcome can be, which may have implications for various aspects of RA treatment strategies.

Keywords: rheumatoid arthritis, tocilizumab, early intervention

\section{Introduction}

Rheumatoid arthritis (RA) is a systemic inflammatory disease of unknown etiology with a prevalence ranging from $0.5 \%$ to $1 \%$ in developed countries. ${ }^{1}$ Although the main target seems to be synovia, nonarticular involvement may occur, which, together with the joint involvement, can lead to major decrements in health-related quality of life, functional limitations, and work ability, and, more importantly, an increased risk of cardiovascular disease that may reduce life expectancy by 3 to 18 years. ${ }^{2}$

The therapeutic landscape in the management of RA has witnessed revolutionary changes over the last 15 years. Glucocorticoids, nonsteroidal anti-inflammatory drugs, and a few disease-modifying anti-rheumatic drugs (DMARDs), including methotrexate (MTX), sulphasalazine, hydroxychloroquine, and gold were the only the treatment options until 2 decades ago. By the invention of new therapeutic targets, a significant breakthrough was achieved in rheumatology with the development of biological agents. The first class of biologic agents to be recognized and used was tumor necrosis factor (TNF) inhibitors, which was followed by many others with different modes of action such as rituximab, abatacept, and tocilizumab (TCZ). These agents have changed the fate of patients with RA due to their good efficacy and safety features. ${ }^{3}$ However, one should keep in mind that it is not only the increased number of available options for clinicians leading to better outcomes but the change in our paradigm while treating patients with RA. One of the major components of our new treatment paradigm is the concept called "window of opportunity," which suggests that disease modification can be optimized by applying intense and effective treatment earlier in the disease course. Indeed, one of the first pieces of evidence supporting this concept originated 
from the analysis of 14 diverse randomized controlled trials conducted with traditional DMARDs, which showed that, regardless of the DMARD used, response to treatment was better when the DMARD was used earlier in the disease course. ${ }^{4}$ This was followed by observations revealing that window of opportunity has also been true and even more robust for TNF inhibitors. Primary results of studies such as Comparison of methotrexate monotherapy with a combination of methotrexate and etanercept in active, early, moderate to severe rheumatoid arthritis (COMET), ${ }^{5}$ PREMIER, ${ }^{6}$ and Behandel-Strategieën study group, (the BeSt study), ${ }^{7}$ as well as subanalyses of the DE019, ${ }^{8}$ Trial of Etanercept and Methotrexate with Radiographic Patient Outcomes (TEMPO), ${ }^{9}$ and ATTRACT ${ }^{10}$ trials, have clearly shown that early institution of TNF inhibitors led to outcomes that were superior to those observed in patients with established disease.

The choice to use TNF inhibitors or biologics with different modes of action is generally reliant on patient and physician preference, as clinical efficacy and safety profiles of these agents are comparable, with very few adverse event profile variances. ${ }^{11}$ While many clinicians prefer to use TNF inhibitors in the first place when biologic agent use is indicated, new options with different modes of action are now available, and some of these may be considered as firstline biologic agents and have been used in this setting with increasing frequency. Despite the increased use of biologics with different modes of action as first-line biologic agents, data regarding their use in early RA are rather scarce compared to TNF inhibitors.

The present article addresses the question of whether a window of opportunity obtained with conventional DMARDs and TNF inhibitors in early RA patients can also be achieved with TCZ. This review therefore aims to highlight the major clinical studies pertaining to the use of TCZ in the management of patients with early RA. There is no consensus about the time interval for defining early RA. While a survey among clinicians defines the patient with early disease as having a disease or symptom duration of less than 3 months, most of the randomized controlled trials accept disease duration of less than 3 years as early RA. ${ }^{12}$ To identify studies to be discussed here, a PubMed search up to June 2013 was performed using the query terms "interleukin-6," "tocilizumab," and "rheumatoid arthritis." Retrieved articles were then assessed and screened for the titles, abstracts, and full texts of the articles as needed to identify relevant studies. We included articles only if the study was setting a limit for disease duration as inclusion criteria ( $<3$ years) and presenting data regarding the subgroup analysis for those patients having early disease, or if the mean disease duration of the study population was less than 3 years. Furthermore, we conducted a similar search in American College of Rheumatology (ACR) and European League Against Rheumatism (EULAR) databases for congress abstracts presented from 2008 to 2013 . We herein review seven studies (five randomized controlled trials and two studies based on the registry data) fulfilling the abovementioned criteria.

\section{TCZ}

TCZ is a fully humanized monoclonal antibody against interleukin (IL)-6 receptors and inhibits the binding of IL-6 to its receptors. IL-6, with its known functions on immune response, acute phase reaction, and hematopoiesis, had been considered as a therapeutic target approximately 2 decades ago. However, it was initially investigated in the field of oncology. Mouse monoclonal antibodies against human IL-6 were found to be effective in a patient with plasma cell leukemia. ${ }^{13}$ Due to the high immunogenic potential of murine antibodies for humans, a humanized form was created and shown to inhibit multiple myeloma cell growth. ${ }^{14}$ On the other hand, TCZ was first approved for Castleman disease, a lymphoproliferative disorder characterized by benign hyperplastic lymph nodes, in 2005. The first case reports demonstrating the success of IL- 6 blockage in this disease were published in the early $1990 \mathrm{~s} .{ }^{15}$ Although the role of IL-6 in the pathogenesis of RA was recognized almost at the same time, the rationale for its use in RA was at least partly dependent on the similarity of symptoms of Castleman disease with RA. ${ }^{16}$ With the results of randomized controlled trials, TCZ was approved for RA first in Japan, then, respectively, in Europe and United States of America. ${ }^{17,18,19}$

\section{Clinical benefits of early intervention with TCZ}

The study of active controlled monotherapy used for rheumatoid arthritis IL-6 inhibitor (SAMURAI) trial was a 1-year, randomized, open-label, but X-ray reader-blinded trial designed to explore whether TCZ monotherapy provides radiographic and clinical benefits to RA patients having an active disease. ${ }^{20}$ Although it was not formally an early intervention study (limitation for disease duration was set as less than 5 years as an eligibility criterion), the mean disease duration of the study population was compatible with the early disease criteria for clinical trials (2.3 years). A total of 306 patients with an inadequate response to at least one DMARD were randomized to either a TCZ monotherapy $(n=158)$ or therapy with conventional DMARDs $(n=148)$. 
Patients in the TCZ group received $8 \mathrm{mg} / \mathrm{kg}$ of TCZ every 4 weeks, while those in the control group received treatment with any conventional DMARDs $(56 \%$ received a combination of MTX and DMARDs, 29\% received MTX monotherapy, and 14\% received DMARDs other than MTX as monotherapy), except for TNF inhibitors or leflunomide. The clinical efficacy of TCZ was better than that found in the DMARD group as assessed by ACR20, ACR50, and ACR70 response rates $(78 \%, 64 \%$, and $44 \%$ versus $34 \%, 13 \%$, and $6 \%$, respectively, $P<0.001$ for each comparison). After 1 year of treatment, clinical remission (defined as disease activity score $[\mathrm{DAS}] 28<2.6$ ) was achieved in $59 \%$ of patients receiving TCZ, compared with $3 \%$ of patients receiving DMARDs $(P<0.001)$. At week 52, 56\% of TCZ-treated patients had no radiographic progression compared to $39 \%$ of those receiving control treatment $(P<0.01)$. Likewise, at the end of the study period, patients in the TCZ group showed statistically less radiographic progression, as measured by the change in total modified Sharp score, than those receiving DMARDs (2.3 versus $6.1 ; P<0.01$ ). The SAMURAI trial also looked at a range of patient-reported outcomes. Significantly more patients receiving TCZ monotherapy achieved a minimum clinically important difference in the modified Health Assessment Questionnaire $(>0.22)$ as compared to the DMARD group $(68 \%$ versus $40 \%, P<0.001)$. The percentage of adverse events was $89 \%$ in the TCZ group but $82 \%$ in the DMARD group, although most were not serious. Overall study results showed that TCZ monotherapy is more effective than DMARD therapies in RA patients with relatively early disease who had received at least one DMARD previously.

In another study, called Chugai humanized anti-Human recombinant interleukin-6 monoclonal antibody (CHARISMA) study, a total of 359 patients were randomized into seven groups consisting of TCZ 2, 4, and $8 \mathrm{mg} / \mathrm{kg}$ as monotherapy; 2, 4, and $8 \mathrm{mg} / \mathrm{kg}$ in combination with MTX; and placebo plus MTX, with an aim of investigating the safety and efficacy of TCZ for patients with an inadequate response to MTX monotherapy. ${ }^{21}$ Although, similar to the SAMURAI trial, short disease duration was not part of the eligibility criteria, the mean disease duration was reported as less than 12 months in all study groups. On the other hand, interpretation of the results of this study as an early RA trial is debatable to some extent, given the fact that the reported mean duration of MTX treatment varied between 29 and 40 months among the study groups. In this 16-week trial, monotherapy with TCZ 4 or $8 \mathrm{mg} / \mathrm{kg}$ was associated with superior ACR20 response rates (primary outcome) in comparison with MTX monotherapy (61\% and $63 \%$ versus $41 \%$, respectively; $P<0.05$ ). In addition, combination with MTX was found to be more efficacious than TCZ monotherapy. In the combination therapy groups, the proportion of patients achieving an ACR20 response was significantly higher in all TCZ doses $(2,4$, or $8 \mathrm{mg} / \mathrm{kg})$ compared with the control group $(64 \%, 63 \%$, and $74 \%$ versus $41 \%$, respectively; $P<0.05, P<0.05$, and $P<0.001$, respectively) at week 16 . With regard to achieving remission at week 16 according to DAS28 score, combination therapy seems to be superior when compared to either TCZ or MTX monotherapy. The rate of remission was $34 \%$ among those assigned to $8 \mathrm{mg} /$ $\mathrm{kg}$ of TCZ plus MTX, 17\% among those receiving $8 \mathrm{mg} / \mathrm{kg}$ of TCZ as monotherapy, and $8 \%$ among those receiving MTX monotherapy. The results of this study clearly indicate that TCZ either as monotherapy or in combination with background MTX can produce marked improvement in disease activity in patients with relatively early disease.

The Actemra versus Methotrexate double-Blind Investigative Trial In mONotherapy (AMBITION) trial was designed to investigate the efficacy and safety of TCZ as monotherapy compared with MTX monotherapy in patients with active RA who had not previously failed either MTX or a biological treatment. ${ }^{22}$ Although the study was not originally designed to evaluate TCZ in early RA (mean disease duration of the study population was 6.3 years), a significant proportion of patients in both arms (nearly 40\%) had disease duration of less than 2 years. It was a 24-week, double-blind, doubledummy randomized trial. A total of 673 patients were recruited to the study, but a subgroup consisting of 101 patients initially treated with placebo for 2 months followed by TCZ were excluded from the efficacy and safety analysis. The remaining 572 patients were randomized to either TCZ $8 \mathrm{mg} / \mathrm{kg}$ every 4 weeks or MTX up to $20 \mathrm{mg} /$ week. The primary endpoint was the proportion of patients with an ACR20 response at week 24. Secondary endpoints included the proportion of patients with ACR50/70 responses at week 24 and the time to onset of ACR20/50/70 responses. At the end of 24 weeks, TCZ was statistically superior to MTX for ACR20, 50, and 70 responses $(70 \%, 45 \%, 27 \%$ versus $54 \%, 34 \%, 15 \%$, respectively; $P$-values for ACR20, 50, and 70 were $0.003,0.01$, and 0.009 , respectively). Post hoc analysis of the AMBITION study revealed that both TCZ (42\% versus $28 \%$ ) and MTX (18\% versus $7 \%$ ) led to significantly higher remission rates in those with RA for less than 2 years compared with those with longer disease duration (both $P<0.05$ ). ${ }^{23}$

Although the participants of the three trials discussed above mainly consisted of patients with early disease, none of the trials was primarily designed to test the efficacy and safety of TCZ in a patient population with early RA. In this year's 
(2013) European League Against Rheumatism (EULAR) meeting, two trials that aimed to study the role of TCZ exclusively in early RA population were presented. ${ }^{24,25}$

The study titled "Tocilizumab in combination and monotherapy versus MTX in MTX-naïve patients with early RA" is a four-arm, double-blinded, double-dummy, randomized, multicenter, Phase III study that was designed to evaluate the efficacy and safety of TCZ alone and in combination with MTX versus MTX monotherapy in patients with early moderate-to-severe RA who had not previously been treated with MTX or a biologic agent. The 1-year results from this 2-year study were presented at EULAR in June 2013. ${ }^{24}$ The study enrolled 1,157 patients who had disease duration of less than 2 years with mean disease duration of 6 months. Patients were randomized to receive TCZ $8 \mathrm{mg} / \mathrm{kg}+\mathrm{MTX}$, TCZ $8 \mathrm{mg} / \mathrm{kg}$ monotherapy, TCZ $4 \mathrm{mg} / \mathrm{kg}+$ MTX or MTX monotherapy. The primary endpoint of the study was the proportion of patients with a DAS28 remission response (DAS28 <2.6) at week 24. Initial randomization to the TCZ $8 \mathrm{mg} / \mathrm{kg}+$ MTX combination was found to be associated with a greater chance of being in remission, as $44.8 \%$ of the patients initially receiving TCZ $8 \mathrm{mg} / \mathrm{kg}$ combination achieved remission at week 24 , compared with $15 \%$ of the patients on MTX monotherapy $(P<0.0001)$. With regard to secondary endpoints, significantly greater proportions of TCZ $8 \mathrm{mg} / \mathrm{kg}+$ MTX than MTX patients achieved ACR20/50/70 responses both at weeks 24 and $52(P<0.05)$. At week 24, $74.5 \%, 56.9 \%$, and $38.6 \%$ ACR20, 50, and 70 responses were seen with TCZ $8 \mathrm{mg} / \mathrm{kg}$ + MTX compared with $65.2 \%$, $43.2 \%$, and $25.4 \%$ in the placebo group, respectively. At week $52,67.2 \%, 55.9 \%$, and $43.1 \%$ ACR20, 50, and 70 responses were seen with TCZ $8 \mathrm{mg} / \mathrm{kg}+$ MTX compared with $57.1 \%$, $40.8 \%$, and $28.9 \%$ in the placebo group, respectively. At 1 year, TCZ $8 \mathrm{mg} / \mathrm{kg}$ + MTX-treated patients showed statistically significant improvement in mean van der Heijde modified total Sharp score. In this trial, there was no difference between the overall incidences of adverse events across the treatment groups.

A similar study by means of design but smaller in scale, Comparative Study of the Clinical Response and Cardiorespiratory Endurance in Early Rheumatoid Arthritis Patients Treated With Tocilizumab or Methotrexate (TOMERA), was also presented at EULAR 2013. ${ }^{25}$ In the TOMERA trial, MTX-naïve patients who had an active disease and disease duration of less than 2 years were studied. A total of 30 patients were randomized to receive either TCZ $8 \mathrm{mg} / \mathrm{kg}$ every month or MTX $20 \mathrm{mg}$ weekly for 6 months. At 6 months, TCZ monotherapy showed higher rates of
DAS28-C-reactive protein remission (76.5\%) compared to MTX treatment (41.7\%), although it did not reach statistical significance $(P=0.1)$. On the other hand, the percentages of patients achieving simple disease activity index $<3.3$ (75\% versus $16.7 \%$ ) and ACR-EULAR Boolean-defined remission $(64.7 \%$ versus $15.4 \%)$ in the TCZ arm were statistically higher than in the MTX arm. The results of this study showed that TCZ monotherapy is more effective in early RA patients by means of achieving remission.

In addition to the randomized controlled trials described above, a few studies based on the patient registry data have focused on the efficacy of TCZ in patients with early RA. Kojima et al analyzed the factors associated with remission in 123 patients treated with TCZ for 52 weeks. ${ }^{26}$ They prospectively followed the patients in Tsurumai Biologics Communication Registry (TBCR), which is a new registry of patients starting treatment with biologics from 2008. The patients with RA were classified according to the disease duration as short, medium, or long ( $\leq 4.8$ years, $4.8-12.0$ years, and $\geq 12.0$ years, respectively). The mean disease duration of patients in the first group $(n=41)$ was 2.2 years, which can be considered as relatively early disease. Although the number of patients who failed TNF inhibitor treatment was similar, the remission rates were significantly higher in patients with short disease duration (31.4\%), in comparison with the medium and long disease durations ( $10.0 \%$ and $2.4 \%$, respectively). In multivariate analysis, along with the lower baseline disease activity, the strongest predictor of remission was short disease duration. Therefore, the authors concluded that remission is an achievable aim for patients treated with TCZ having short disease duration. Although the number of patients was limited, the data are important, since they reflect the actual clinical practice.

In a recently published study from the same registry, the investigators further analyzed the efficacy of TCZ when used as a first-line biological drug for RA patients with short disease duration. ${ }^{27}$ In the same cohort (TBCR), they focused on a total of 50 patients who had received TCZ as a first-line treatment, and divided the original cohort into two groups according to disease duration at baseline of less than or more than 12 months. The number of patients in each group was 10 and 40, respectively. Both the conventional and new Boolean-based remission criteria were used for analysis. ${ }^{28}$ After 12 months' treatment, according to the new remission criteria, the rate of remission was higher in patients with early disease than in those with longer disease $(50 \%$ versus $12.5 \%$ ), although there was no difference with respect to the conventional criteria. 


\section{Conclusion}

According to the data presented here, TCZ has been proven to be effective in patients with early RA, either as combination therapy with MTX or as monotherapy, while the clinical response is much better when it is used in combination. Although all the studies investigating TCZ treatment in early RA patients showed superior clinical and radiological response compared to placebo, the question of whether treating patients earlier with TCZ is more advantageous than instituting this agent in patients with established disease is difficult to answer. The unequivocal solution to this question can only be achieved by conducting studies testing both strategies (early versus late treatment with TCZ) in the same trial, similarly to previously conducted studies such as BeST. ${ }^{7}$ The alternative but less reliable approach to this problem is to compare clinical response rates between the studies conducted in early versus established RA patients, although this is very challenging because of the lack of consistency with regard to design between the studies. Both the OPTION $^{29}$ and TOWARD ${ }^{30}$ studies were conducted among established RA patients who showed incomplete response to DMARDs. The studies conducted in early RA patients corresponding to OPTION and TOWARD studies are SAMURAI ${ }^{20}$ and CHARISMA. ${ }^{21}$ When we compared ACR20 responses between the studies conducted in early and established disease, the proportion of patients achieving this response was numerically higher in patients with early disease (74\%-78\%) compared to those with established disease (60\%). Despite the higher rate of response observed among the patients with early disease, one should keep in mind that the floor effect (ie, patients with more damage have less potential for improvement, even if their disease activity improves the same amount) might be responsible, at least partly, for this observation. Considering the studies mentioned in this review, efficacy outcomes for TCZ in patients with early RA seem not to be different from those of the studies conducted with TNF inhibitors, while no head-to-head comparative trials exist. Indeed, treatment with TCZ, particularly early in the disease course, might be associated with better outcomes in the long-term, considering the potential beneficial effects that can be achieved by blockage of IL- 6 .

There has been evidence to suggest that IL- 6 is one of the key cytokines in the pathogenesis of RA. In the late 1980s, the levels of IL- 6 were reported to be elevated both in the sera and synovial fluid of RA patients. ${ }^{31}$ These findings suggest that IL-6 not only has a local but also a systemic role in the development of inflammatory arthritis. The synovitis in RA is characterized by the synovial hyperplasia, as well as by inflammatory cell infiltration and angiogenesis. The key molecules that have been implicated in rheumatoid angiogenesis are vascular endothelial growth factor (VEGF) and angiopoietins. In a study by Nakahara et al, anti-IL-6 receptor antibody treatment was reported to decrease the serum VEGF levels. ${ }^{32}$ Most recently, it has been demonstrated that IL-6 increases VEGF expression and inhibits Angiopoietin-1. ${ }^{33}$ Since angiogenesis contributes not only to pannus formation but also bone and cartilage destruction, these data confirm the central role for IL- 6 in RA. ${ }^{34}$ The contribution of IL-6 to joint erosion is not limited to angiogenesis only, since it has been found to be involved in osteoclast stimulation and matrix metalloproteinase production. ${ }^{35,36}$ Considering the abovementioned pathogenic role of IL-6 in RA, particularly in the progression of joint damage, blockage of this cytokine early in the disease course might have additional benefits that can slow or even stop the disease progression.

In conclusion, on the basis of currently available data, it is reasonable to accept the claim that the earlier the TCZ treatment, the better the clinical outcome can be.

\section{Disclosure}

The authors report no conflicts of interest in this work.

\section{References}

1. Gabriel SE, Michaud K. Epidemiological studies in incidence, prevalence, mortality, and comorbidity of the rheumatic diseases. Arthritis Res Ther. 2009; 11(3):229.

2. Pincus T, Kavanaugh A, Sokka T. Benefit/risk of therapies for rheumatoid arthritis: underestimation of the "side effects" or risks of RA leads to underestimation of the benefit/risk of therapies. Clin Exp Rheumatol. 2004;22(5) Suppl 35:2-11.

3. Nam JL, Winthrop KL, van Vollenhoven RF, et al. Current evidence for the management of rheumatoid arthritis with biological diseasemodifying antirheumatic drugs: a systematic literature review informing the EULAR recommendations for the management of RA. Ann Rheum Dis. 2010;69(6):976-986

4. Anderson JJ, Wells G, Verhoeven AC, Felson DT. Factors predicting response to treatment in rheumatoid arthritis: the importance of disease duration. Arthritis Rheum. 2000;43:22-29.

5. Emery P, Breedveld FC, Hall S, et al. Comparison of methotrexate monotherapy with a combination of methotrexate and etanercept in active, early, moderate to severe rheumatoid arthritis (COMET): a randomised, double-blind, parallel treatment trial. Lancet. 2008;372:375-382.

6. Breedveld FC, Weisman MH, Kavanaugh AF, et al. The PREMIER study: a multicenter, randomized, double-blind clinical trial of combination therapy with adalimumab plus methotrexate versus methotrexate alone or adalimumab alone in patients with early, aggressive rheumatoid arthritis who had not had previous methotrexate treatment. Arthritis Rheum. 2006;54:26-37.

7. Goekoop-Ruiterman YP, de Vries-Bouwstra JK, Allaart CF, et al. Clinical and radiographic outcomes of four different treatment strategies in patients with early rheumatoid arthritis (the BeSt study): a randomized controlled trial. Arthritis Rheum. 2005;52:3381-3390.

8. Jamal S, Patra K, Keystone EC. Adalimumab response in patients with early versus established rheumatoid arthritis: DE019 randomized controlled trial subanalysis. Clin Rheumatol. 2009;28(4):413-419. 
9. Gómez-Reino J. Biologic monotherapy as initial treatment in patients with early rheumatoid arthritis. Rheumatology (Oxford). 2012;51 Suppl 5: v31-v37.

10. Keystone EC, Haraoui B, Bykerk VP. Role of infliximab in the treatment of early rheumatoid arthritis. Clin Exp Rheumatol. 2003; 21(5) Suppl 31:200-202.

11. Yazici Y, McMorris BJ, Darkow T, Rosenblatt LC. Patient and physician perception of the infusion process of the biologic agents abatacept, infliximab, and rituximab for the treatment of rheumatoid arthritis. Clin Exp Rheumatol. 2009;27:907-913.

12. Aletaha D, Eberl G, Nell VP, Machold KP, Smolen JS. Attitudes to early rheumatoid arthritis: changing patterns. Results of a survey. Ann Rheum Dis. 2004;63(10):1269-1275.

13. Klein B, Wijdenes J, Zhang XG, Jourdan M, Boiron JM, Brochier J. Murine anti-interleukin- 6 monoclonal antibody therapy for a patient with plasma cell leukemia. Blood. 1991;78:1198-1204.

14. Sato K, Tsuchiya M, Saldanha J, et al. Reshaping a human antibody to inhibit the interleukin 6-dependent tumor cell growth. Cancer Res. 1993;53(4):851-856.

15. Beck JT, Hsu SM, Wijdenes J, et al. Brief report: alleviation of systemic manifestations of Castleman's disease by monoclonal anti-interleukin-6 antibody. N Engl J Med. 1994;330:602-605.

16. Sack U, Kinne RW, Marx T, Heppt P, Bender S, Emmrich F. Interleukin-6 in synovial fluid is closely associated with chronic synovitis in rheumatoid arthritis. Rheumatol Int. 1993;13(2):45-51.

17. Pharmaceuticals and Medical Devices Agency [homepage on the internet]. Tokyo, Japan. Available from http://www.pmda.go.jp/english/ service/pdf/drugs/actemra_apr2008_e.pdf. Accessed September 30, 2013.

18. European Medicines Agency [homepage on the internet]. London. Available from http://www.ema.europa.eu/docs/en_GB/document_library/ EPAR_-_Public_assessment_report/human/000955/WC500054888. pdf/. Accessed September 30, 2013.

19. U.S. Food and Drug Administration [homepage on the internet]. USA. Available from http://www.accessdata.fda.gov/drugsatfda_docs/ nda/2010/125276s000TOC.cfm. Accessed September 30, 2013.

20. Nishimoto N, Hashimoto J, Miyasaka N, et al. Study of active controlled monotherapy used for rheumatoid arthritis, an IL-6 inhibitor (SAMURAI): evidence of clinical and radiographic benefit from an $\mathrm{x}$ ray reader-blinded randomised controlled trial of tocilizumab. Ann Rheum Dis. 2007;66:1162-1167.

21. Maini RN, Taylor PC, Szechinski J, et al. Double-blind randomized controlled clinical trial of the interleukin-6 receptor antagonist, tocilizumab, in European patients with rheumatoid arthritis who had an incomplete response to methotrexate. Arthritis Rheum. 2006;54(9):2817-2829.

22. Jones G, Sebba A, Gu J, et al. Comparison of tocilizumab monotherapy versus methotrexate monotherapy in patients with moderate to severe rheumatoid arthritis: the AMBITION study. Ann Rheum Dis. 2010;69:88-96.

23. Jones G. The AMBITION trial: tocilizumab monotherapy for rheumatoid arthritis. Expert Rev Clin Immunol. 2010;6(2):189-195.

24. Burmester G, Rigby W, Vollenhoven RV, et al. Tocilizumab in combination and monotherapy versus methotrexate in methotrexate-naive patients with early rheumatoid arthritis: clinical and radiographic outcomes from a randomised, placebo-controlled trial. Ann Rheum Dis. 2013;72(Suppl 3):63.
25. Durez P, Depresseux G, Toukap AN, et al. Rate of remission by tocilizumab or methotrexate induction therapy in early active rheumatoid arthritis: results of the TOMERA trial. Ann Rheum Dis. 2013; 72(Suppl 3):623.

26. Smolen JS, Beaulieu A, Rubbert-Roth A, et al. Effect of interleukin-6 receptor inhibition with tocilizumab in patients with rheumatoid arthritis (OPTION study): a double-blind, placebo-controlled, randomised trial. Lancet. 2008:22;371(9617):987-997.

27. Genovese MC, McKay JD, Nasonov EL, et al. Interleukin-6 receptor inhibition with tocilizumab reduces disease activity in rheumatoid arthritis with inadequate response to disease-modifying antirheumatic drugs: the tocilizumab in combination with traditional disease-modifying antirheumatic drug therapy study. Arthritis Rheum. 2008;58(10):2968-2980.

28. Kojima T, Kaneko A, Hirano Y, et al. Early aggressive intervention with tocilizumab for rheumatoid arthritis increases remission rate defined using a Boolean approach in clinical practice. Mod Rheumatol. 2012;22(3):370-375.

29. Yabe Y, Kojima T, Kaneko A, Asai N, Kobayakawa T, Ishiguro N. A review of tocilizumab treatment in 122 rheumatoid arthritis patients included in the Tsurumai Biologics Communication Registry (TBCR) Study. Mod Rheumatol. 2013;23(2):245-253.

30. Felson DT, Smolen JS, Wells G, et al; American College of Rheumatology; European League Against Rheumatism. American College of Rheumatology/European League Against Rheumatism provisional definition of remission in rheumatoid arthritis for clinical trials. Arthritis Rheum. 2011;63:573-586.

31. Houssiau FA, Devogelaer JP, van Damme J, de Deuxchaisnes CN, Van Snick J. Interleukin-6 in synovial fluid and serum of patients with rheumatoid arthritis and other inflammatory arthritides. Arthritis Rheum. 1988:31:784-788.

32. Nakahara H, Song J, Sugimoto M, et al. Anti-interleukin-6 receptor antibody therapy reduces vascular endothelial growth factor production in rheumatoid arthritis. Arthritis Rheum. 2003;48:1521-1529.

33. Kayakabe K, Kuroiwa T, Sakurai N, et al. Interleukin-6 promotes destabilized angiogenesis by modulating angiopoietin expression in rheumatoid arthritis. Rheumatology (Oxford). 2012;51(9):1571-1579.

34. Firestein GS. Starving the synovium: angiogenesis and inflammation in rheumatoid arthritis. J Clin Invest. 1999;103:3-4.

35. Palmqvist P, Persson E, Conaway HH, Lerner UH. IL-6, leukemia inhibitory factor, and oncostatin $\mathrm{M}$ stimulate bone resorption and regulate the expression of receptor activator of NF-kappa B ligand, osteoprotegerin, and receptor activator of NF-kappa B in mouse calvariae. J Immunol. 2002;169(6):3353-3362.

36. Karsdal MA, Schett G, Emery P, et al. IL-6 receptor inhibition positively modulates bone balance in rheumatoid arthritis patients with an inadequate response to anti-tumor necrosis factor therapy: biochemical marker analysis of bone metabolism in the tocilizumab RADIATE study (NCT00106522). Semin Arthritis Rheum. 2012;42(2):131-139.
Therapeutics and Clinical Risk Management

\section{Publish your work in this journal}

Therapeutics and Clinical Risk Management is an international, peerreviewed journal of clinical therapeutics and risk management, focusing on concise rapid reporting of clinical studies in all therapeutic areas, outcomes, safety, and programs for the effective, safe, and sustained use of medicines. This journal is indexed on PubMed Central, CAS,

\section{Dovepress}

EMBase, Scopus and the Elsevier Bibliographic databases. The manuscript management system is completely online and includes a very quick and fair peer-review system, which is all easy to use. Visit $\mathrm{http}: / / \mathrm{www}$. dovepress.com/testimonials.php to read real quotes from published authors. 